\title{
A new comprehensive gene expression panel to study tumor micrometastasis in patients with high-risk breast cancer
}

\author{
P.F. FERRUCCI, C. RABASCIO, F. GIGLI, C. CORSINI, \\ G. GIORDANO, F. BERTOLINI and G. MARTINELLI \\ Division of Hemato-oncology, European Institute of Oncology, Milan, Italy
}

Received October 3, 2006; Accepted November 27, 2006

\begin{abstract}
The incidence and prognostic relevance of bone marrow (BM) and leukapheresis (PBPC) tumor cell contamination (TCC) in breast cancer patients is still to be circumstantiated. We developed a new comprehensive gene expression panel to study cytokeratins (CK), maspin (MAS) and mammaglobin (MAM) as possible predictors of prognosis. Forty-eight patients undergoing high dose chemotherapy (HDCT) and PBPC support were enrolled and analyzed for TCC on 116 PBPC apheresis and 96 BM obtained at basal conditions. All of the patients were evaluated by reverse transcriptase nested PCR (RT-PCR) for MAM and MAS gene expression and by immunocytochemistry (ICC) and nested RT-PCR to evaluate CK expression. PBPC and BM frequency of CK-positive (+) cells was $12-13 \%$ by ICC and $71-73 \%$ by RT-PCR respectively. Sixty-seven percent of CK $\mathrm{ICC}^{+}$samples were MAM RT-PCR ${ }^{+}$and $89 \%$ of them were MAS RT-PCR ${ }^{+}$. PBPC and BM frequency of $\mathrm{MAM}^{+}$cells was $21 \%$ and $31 \%$ respectively, while for $\mathrm{MAS}^{+}$cells it was $48 \%$ and $52 \%$ respectively by RT-PCR. After 71 mo median FU, 16 patients $(33 \%)$ relapsed and $14(88 \%)$ had BM/PBPC TCC. No marker had an impact on overall survival (OS) but MAS expression on BM and MAM expression on PBPC correlated with a statistically significant improved $(\mathrm{p}=0.05)$ and worsened RFS ( $\mathrm{p}=0.06$ ) respectively. These data confirm the activity of MAM as a negative prognostic factor and show for the first time that MAS could work as a tumor suppressor gene even in a clinical setting, since it protects from recurrence.
\end{abstract}

\section{Introduction}

The reinfusion of mobilized PBSC has become the standard procedure in autologous transplantation after HDCT for

Correspondence to: Dr Pier Francesco Ferrucci, Division of Hemato-oncology, European Institute of Oncology, via Ripamonti 435, I-20141 Milan, Italy

E-mail: pier.ferrucci@ieo.it

Key words: micrometastases, bone marrow, breast cancer, peripheral blood progenitor cells, cytokeratins, maspin different neoplastic diseases. This is supported by a high number of phase II-III clinical trials, showing that patients treated with this stem cell source engraft more rapidly than those treated with BM (1). HDCT with hematopoietic support produces high response rates and some long-term diseasefree survivors, in patients with metastatic breast carcinoma (2). Moreover, HDCT treatment in patients with high-risk breast cancer appears to improve relapse-free survival (RFS) and overall survival (OS) if compared to standard CT at least in a randomized trial (3), but it is still a matter of debate.

As many questions still remain unanswered regarding the best methods to detect the presence of tumor cells in peripheral blood, BM and/or apheresis products, the impact of BM and leukapheresis tumor cell contamination (TCC) on patient prognosis and therapeutic outcome must be circumstantiated. However, TCC is common in PBSC samples, raising the issue of whether current techniques are able to identify, reduce, remove or separate them from the stem samples.

Between 4 and $50 \%$ of women with primary breast cancer have tumor cells contaminating their bone marrow at the time of diagnosis, as determined by sensitive immunocyto-chemical techniques $(4,5)$. Some investigators correlated micrometastatic disease in the bone marrow with other prognostic factors and determined micrometastatic disease in the bone marrow to be an independent predictor of relapse (6-8).

Other authors suggest that tumor cells in nodes and/or in bone marrow may represent different populations of metastatic clones $(7,9)$. On the other hand, there is now some evidence that TCC of stem cell products may be related to disease relapse, since Brenner and colleagues, demonstrated that previously gene-marked tumor cells, after reinfusion contributed to neoplastic recurrence (10).

Many studies show a correlation between BM TCC and OS (Moss, et al, Proc ASCO 16: abs. 90a, 1997; 11-18), but metaanalyses on 20 studies regarding a total of 2,494 patients, failed to clearly demonstrate the impact of BM micrometastatic disease on prognosis. In particular 5 out of 11 studies indicated the presence of epithelial cells in the BM as an independent predictor of short disease-free survival (DFS) and only 2 out of 12 showed a correlation with OS using multivariate analysis. In conclusion meta-analyses confirm the need for further studies using standardized protocols before micrometastasis detection can be used as a prognostic tool for use in the TNM classification (19).

More specific markers are needed to better understand the real impact of TCC on prognosis of breast cancer patients: 
Table I. Characteristics of the patients enrolled in the study.

\begin{tabular}{|c|c|c|c|c|c|c|c|}
\hline Patients & Start & Stage & $>20 \mathrm{LN}^{+}$ & Schedule & Relapse & Site & Follow-up \\
\hline 1 & 10/08/98 & IIIA & no & T-ICE & $18 / 12 / 00$ & bone & $14 / 05 / 02$ \\
\hline 2 & $12 / 08 / 98$ & IIB & no & $\mathrm{EC}$ & 13/06/01 & bone & 03/07/02 \\
\hline 3 & $25 / 08 / 98$ & IIB & no & $\mathrm{T}-\mathrm{EC}$ & no & & $14 / 02 / 01$ \\
\hline 4 & $31 / 08 / 98$ & IIB & no & T-EC & no & & $16 / 04 / 02$ \\
\hline 5 & $15 / 09 / 98$ & IIB & no & $\mathrm{EC}$ & no & & $12 / 06 / 02$ \\
\hline 6 & 28/09/98 & IIB & no & T-ICE & 12/05/99 & breast & dead \\
\hline 7 & 28/09/98 & IIIA & yes & T-EC & 08/09/99 & liver & $20 / 05 / 02$ \\
\hline 8 & 05/10/98 & IIA & no & $\mathrm{EC}$ & $16 / 11 / 00$ & lung and liver & 05/04/02 \\
\hline 9 & $12 / 10 / 98$ & IIB & no & T-ICE & $01 / 12 / 99$ & brain & dead \\
\hline 10 & $02 / 11 / 98$ & IIIA & no & T-ICE & $22 / 12 / 98$ & abdomen & dead \\
\hline 11 & $11 / 11 / 98$ & IIA & no & $\mathrm{T}-\mathrm{EC}$ & no & & 20/02/02 \\
\hline 12 & $16 / 11 / 98$ & IIB & no & T-EC & 16/09/99 & brain & dead \\
\hline 13 & $16 / 11 / 98$ & IIB & no & T-EC & no & & $19 / 04 / 02$ \\
\hline 14 & $17 / 11 / 98$ & IB & no & $\mathrm{EC}$ & 10/05/99 & cervical LN & 07/06/01 \\
\hline 15 & $17 / 11 / 98$ & IIIA & yes & T-EC & no & & $15 / 04 / 02$ \\
\hline 16 & 23/11/98 & IIIA & yes & T-EC & no & & 08/07/02 \\
\hline 17 & $23 / 11 / 98$ & IIB & no & T-EC & $01 / 11 / 99$ & bone & dead \\
\hline 18 & $25 / 11 / 98$ & IIB & no & $\mathrm{T}-\mathrm{EC}$ & no & & 05/06/02 \\
\hline 19 & $10 / 12 / 98$ & IIB & no & T-ICE & 20/05/99 & skin & dead \\
\hline 20 & $14 / 12 / 98$ & IIB & no & $\mathrm{EC}$ & no & & $10 / 04 / 02$ \\
\hline 21 & $14 / 12 / 98$ & IIB & no & $\mathrm{T}-\mathrm{EC}$ & no & & $16 / 06 / 02$ \\
\hline 22 & 13/01/99 & IIIA & no & ICE & $01 / 02 / 00$ & lung and brain & dead \\
\hline 23 & 18/01/99 & IIB & no & T-EC & $26 / 04 / 02$ & lung and LN SC & $11 / 07 / 02$ \\
\hline 24 & 25/01/99 & IIIA & yes & $\mathrm{EC}$ & no & & $17 / 04 / 02$ \\
\hline 25 & 04/02/99 & IIB & no & $\mathrm{EC}$ & no & & $19 / 04 / 02$ \\
\hline 26 & $04 / 02 / 99$ & IIB & no & T-EC & no & & $22 / 05 / 02$ \\
\hline 27 & 22/02/99 & IIB & no & T-EC & $16 / 02 / 01$ & liver & $10 / 05 / 02$ \\
\hline 28 & 01/03/99 & IIA & yes & T-EC & no & & $30 / 01 / 02$ \\
\hline 29 & 01/03/99 & IIB & yes & T-EC & $01 / 03 / 02$ & bone & $14 / 06 / 02$ \\
\hline 30 & 01/03/99 & IIIA & yes & $\mathrm{T}-\mathrm{EC}$ & 28/12/99 & liver & dead \\
\hline 31 & 01/03/99 & IIIA & yes & ICE & 10/10/01 & LN clavear & $11 / 03 / 02$ \\
\hline 32 & 03/03/99 & II & no & $\mathrm{EC}$ & $01 / 02 / 02$ & liver & dead \\
\hline 33 & 08/03/99 & IIB & no & $\mathrm{EC}$ & no & & $10 / 06 / 02$ \\
\hline 34 & 15/03/99 & IIIB & no & ICE & 28/09/99 & liver and breast & dead \\
\hline 35 & 15/03/99 & IIIA & yes & T-EC & $30 / 01 / 01$ & brain & dead \\
\hline 36 & 18/03/99 & IIB & no & T-EC & no & & $31 / 01 / 02$ \\
\hline 37 & 06/04/99 & IIB & no & T-EC & 12/11/01 & skin & 06/03/02 \\
\hline 38 & $17 / 05 / 99$ & IIB & no & $\mathrm{EC}$ & no & & $16 / 01 / 02$ \\
\hline 39 & 28/06/99 & IIB & no & T-EC & no & & $18 / 05 / 00$ \\
\hline 40 & 05/07/99 & IIB & no & $\mathrm{EC}$ & no & & $09 / 05 / 02$ \\
\hline 41 & 06/07/99 & IIB & no & T-EC & no & & $15 / 05 / 02$ \\
\hline 42 & 03/08/99 & IIIA & no & ICE & no & & $18 / 03 / 02$ \\
\hline 43 & 09/08/99 & IIB & no & T-EC & $31 / 01 / 02$ & brain & $23 / 05 / 02$ \\
\hline 44 & 10/08/99 & IIB & no & T-EC & no & & 18/04/02 \\
\hline 45 & 16/08/99 & IIIA & no & T-EC & no & & $17 / 06 / 02$ \\
\hline 46 & 25/08/99 & IIIA & no & ICE & no & & 02/08/01 \\
\hline 47 & 06/09/99 & IIA & no & $\mathrm{EC}$ & no & & 19/06/02 \\
\hline 48 & 07/09/99 & IIIA & no & T-EC & 05/05/00 & lung and liver & dead \\
\hline
\end{tabular}

Patients received 3 cycles of myeloablative chemotherapy followed by a reinfusion of autologous hematopoietic progenitors. TEC $=$ Taxotere + Epirubicin + Cyclofosphamide; T-ICE = Taxotere + Ifosphamide + Carboplatin + Etoposide, EC = Epirubicin + Cyclofosphamide; ICE $=$ Ifosphamide + Carboplatin +Etoposide. 
maspin and mammaglobin might represent good candidates to type this disease.

Maspin is a protein related to the serpin family of protease inhibitors and is widely expressed in epithelial tissues. In vitro it shows tumor suppressor activity, acts directly on endothelial cells to stop their migration towards b-fibroblastic growth factor (b-FGF) and vascular endothelial growth factor (VEGF) treatment and limits mitogenesis and tube cellular formation. In a xenograft mouse model of human prostate cancer, maspin blocks tumor growth and reduces tumor-associated microvessel density working as an anti-angiogenesis modulator $(20,21)$. Moreover, Xia and colleagues demonstrated that maspin expression is linked with improved survival of patients with oral squamous cell carcinoma being associated with reduced lymph-nodal involvement and favorable prognosis (22). Shi and colleagues demonstrated in a syngeneic breast cancer mouse model that overexpression of the maspin gene is able to block tumor growth, invasion and metastasis, supporting the concept of a protein with a strong protective role against tumor progression (23).

Mammaglobin, a mammary-specific member of the uteroglobin gene family is a glicoprotein overexpressed in human breast cancer (24). The expression seems to be related to progression from localized to locally advanced and metastatic disease (25).

The present study was designed to answer the pending questions about the significance of TCD in the BM and its correlation with TCC of the apheresis using different standard and innovative markers.

\section{Patients and methods}

Patient characteristics. Forty-eight patients were enrolled in the study from September 1998 to September 1999. Informed consent was obtained according to the Helsinki Declaration. Patients' ages ranged between 16 and 65 years. Each had a performance status of 0-1 (ECOG scale), histologically proven breast cancer and underwent surgery followed by adjuvant HDCT with PBPC support.

The primary tumor was classified as T1-T3, N1-2, or M0, (UICC 1993) and the patients entered at least one of the following categories: a) $\geq 10$ involved axillary nodes; b) $\geq 5$ involved axillary nodes and ER-negative primary tumor; c) $\geq 5$ involved axillary nodes and T3 primary tumor. Characteristics of the patients are reported in Table I.

Sample harvesting and processing. Forty-eight patients were analyzed for TCC on PBPC apheresis and BM. BM aspiration was performed under local anesthesia from posterior iliac crest puncture before chemotherapy delivery. Five to ten milliliters of aspirate was collected and lysed by $\mathrm{NH}_{4} \mathrm{Cl}$ to remove red cells, resuspended in buffered saline, washed twice

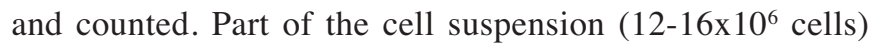
was cytospinned on slides and the rest stored with guanidine isothiocyanate at $-80^{\circ} \mathrm{C}$ for further RNA analysis.

Immunocytochemical staining. Slides were stained with a commercially available alkaline phosphatase anti-alkaline phosphatase (APAAP) based kit for $\mathrm{CK}^{+}$breast cancer cell detection. The identification of epithelial cells is based on the reactivity of murine monoclonal antibody A45-B/B3 with the epithelial cell cytoskeleton, which is mainly composed of cytokeratins. Cells from peripheral blood (PB), PBPC or BM are of mesenchymal origin and their cytoskeleton is mainly composed of vimentin and thus it does not react with the antibody. A range of 9-12x $10^{6}$ cells per sample was analyzed by microscopy for the enumeration of breast cancer cells. Peripheral blood nucleated cells from healthy donors were contaminated with $5 \%$ breast cancer cell line MCF-7 and used as the positive control of contamination.

Reverse transcriptase-polymerase chain reactions. Total cellular RNA was extracted from cells by QIAamp RNA kit (Qiagen, Hilden, Germany) and treated with a reverse transcriptase enzyme (Super Script II, Gibco, Gaithersburg, MD). The obtained cDNA was amplified for CK, MAM and MAS expression according to Mochinsky et al (26), Zach et al (27) and Luppi et al (28), respectively. Single round RT-PCR using $\mathrm{B}$-actin specific primers confirmed the presence of intact RNA, an adequate cDNA synthesis as well as the absence of inhibitors. Amplified products for CK, MAM and MAS were revealed by single bands of 108-201-175 base pairs respectively on ethidium bromide-stained $2 \%$ agarose gel. To reduce false positives for CK transcript we introduced a hybridization step with an internal radiolabelled probe as previously described (41).

Statistical analysis. Progression-free time was calculated from the start of therapy to the date of disease progression. Patients who did not have any progression were censored at their last follow-up time. The progression-free survival was estimated by the Kaplan and Meier method and the log-rank test was used to test for differences between groups of patients.

\section{Results}

\section{Specificity and sensitivity}

CK hybridization with an internal probe to avoid false positive results. Specificity was tested on 12 cell lines, 13 BM of patients affected by non-epithelial malignancies, 12 $\mathrm{BM}$ and PB of healthy donors (Table II). Specificity of the CKamplified samples was confirmed by hybridization with a specific and ${ }^{32} \mathrm{P}$-labeled internal probe, showing that not all the RT-PCR amplified products were indeed CK19 transcripts. In fact about $30 \%$ of them did not anneal to the probe and did not reveal themselves by autoradiography (41). To determine the RT-PCR sensitivity of the three markers, PBMCs obtained from donors were mixed to decreasing numbers of MCF7 and CG-5 breast cancer cell lines. Sensitivity of CK, MAM and MAS methods was $1 \times 10^{-7}, 1 \times 10^{-6}$, and $1 \times 10^{-6}$ cells respectively.

No false positive results were detected in cell lines, apart for the K-562 leukemic cell line, which expressed MASRNA. Moreover, no false positives were found in BM and PB samples used as controls of specificity, in any of the amplification procedures for CK, MAM and MAS as reported in Table II.

$C K$ expression revealed by ICC and nested RT-PCR. By ICC, cells were easily distinguishable from background mononuclear 
Table II. Controls of specificity for CK, MAM and MAS expression.

\begin{tabular}{lcccc}
\hline Controls of specificity & No. & CK (RT-PCR) & MAM (RT-PCR) & MAS (RT-PCR) \\
\hline Healthy donors BMs and PB & 12 & Negative & Negative & Negative \\
Non-epithelial malignancies BMs & 13 & Negative & Negative & Negative \\
Cell lines & 12 & Negative & Negative & K-562 positive \\
\hline
\end{tabular}

Controls of specificity for CK, MAM and MAS expression on 12 healthy donor bone marrow (BM) and peripheral blood samples (PB), 13 bone marrow samples of patients affected by on-epithelial malignancies and 12 cell lines (TF1, CEM, Namalwa, Molt, Huvec, K-562, Sultan Neo, Rap1, Dohh2, Karpass 299, Bonna 12, Jurcatt).

Table III. Number of patients with positive samples for each method and sample is reported in total numbers and percentage respectively.

\begin{tabular}{lcccc}
\hline Samples & $\begin{array}{c}\text { CK } \\
(\text { IHC) }\end{array}$ & $\begin{array}{c}\text { CK } \\
(\text { RT-PCR) }\end{array}$ & $\begin{array}{c}\text { MAS } \\
\text { (RT-PCR) }\end{array}$ & $\begin{array}{c}\text { MAM } \\
\text { (RT-PCR) }\end{array}$ \\
\hline BM & $5 / 48$ & $35 / 48$ & $25 / 48$ & $15 / 48$ \\
& $(12 \%)$ & $(73 \%)$ & $(52 \%)$ & $(31 \%)$ \\
PBPC & $6 / 48$ & $34 / 48$ & $23 / 48$ & $10 / 48$ \\
& $(13 \%)$ & $(71 \%)$ & $(48 \%)$ & $(21 \%)$ \\
\hline
\end{tabular}

cells by bright red staining of cell membrane and cytoplasm. On 116 PBPC and 96 BM specimens, $\mathrm{CK}^{+}$cells were detected in $6 / 48$ patients $(13 \%)$ and in $5 / 48$ patients $(12 \%)$ respectively (Table III).

Using a nested RT-PCR assay for CK19 followed by specific probe hybridization and autoradiography, a single 175-bp band was identifiable in a much higher percentage of cases, without loosing specificity. Results showed CK19 amplification on PBPC specimens of $34 / 48$ patients (71\%), and on BM of 35/48 patients (73\%) (Table III).

Southern blotting with a radiolabelled CK-specific probe after PCR amplification, reduced false positive results by $30 \%$ (41), demonstrating that pseudogene expression and/ or illegitimate transcription represent a real but avoidable problem.

MAM expression and its relationship with $C K$ expression. Results are reported in Table III and show 10/48 positive samples $(21 \%)$ on PBPC and 15/48 (31\%) on BM. We found a good correlation as expressed by Pearson's coefficent between ICC evaluation for CK and RT-PCR for MAM, since all the ICC $\mathrm{CK}^{+}$specimens resulted in an overexpression of the MAM transcript, showing a high reliability of the two methods. No correlation existed when the comparison involved the MAS gene evaluation.

MAS expression and its relationship with CK expression. Fortyeight percent of the patients (23/48) were found to be MAS positive when evaluated on PBPC samples, while $52 \%$ of them (25/48) expressed the MAS product on BM, with an inverse relationship when compared to disease relapses (Table III).
Results obtained with the amplification of the MAS gene in the chosen high-risk breast cancer patient group did not show a good correlation with CK amplification or immunostaining and even with MAM expression as already reported by Lopez-Guerrero et al (29).

\section{RFS and OS on the basis of tumor cell detection in BM and/or} $P B P C$

MAS expression protects from disease relapse, while MAM is a marker of poor prognosis. Studying our results on PBPC collections and BM, we tested all of the possible combinations, matching the expression of the 3 markers, CK, MAM and MAS with patient relapses in order to score a possible effect on prognosis. Not one case was found to have a statistically significant correlation between OS in those patients who had tumor cell detection in BM and/or PBSC versus those who do not, independently for the marker or the method used. When RFS was analyzed, MAS expression on BM specimens and MAM expression on PBPC seemed to impact on the probability of relapse (Figs. 1 and 2).

Sixteen out of $48(33 \%)$ patients relapsed in the subset of PBPC and/or BM samples: 4/16 (25\%) had positive CK ICC staining, 14/16 (87.5\%) had positive CK PCR amplification, $8 / 16(50 \%)$ had positive MAM PCR amplification and 8/16 (50\%) had positive MAS PCR amplification respectively (Table III).

After a median follow-up of 71 months, differences between RFS and OS curves were not statistically significant for CK as a marker of occult micrometastasis either in the bone marrow or in PBPC collections when evaluated either by PCR or ICC (Fig. 1).

Starting at two years, PBPC MAM-positive vs -negative curves on RFS showed a clear trend of diversion, which became nearly statistically significant $(p=0.06)$ with longer follow-up confirming our previously reported data (41). On the other hand, MAS expression identified a subset of patients who showed a statistically significant lower relapse rate and thus a better RFS $(p=0.05)$. Significance was reached only when we evaluated RFS on BM but neither on PBPC alone or in combination with BM, nor for OS probably due to the low numbers of events reported (Fig. 2).

Interestingly, among the patients found positive for $\mathrm{CK}$ and MAM expression in at least one sample, which were considered micrometastatic, the contemporary expression of MAS protects from relapse and patients have a statistically significant advantage concerning DFS (data not shown). 

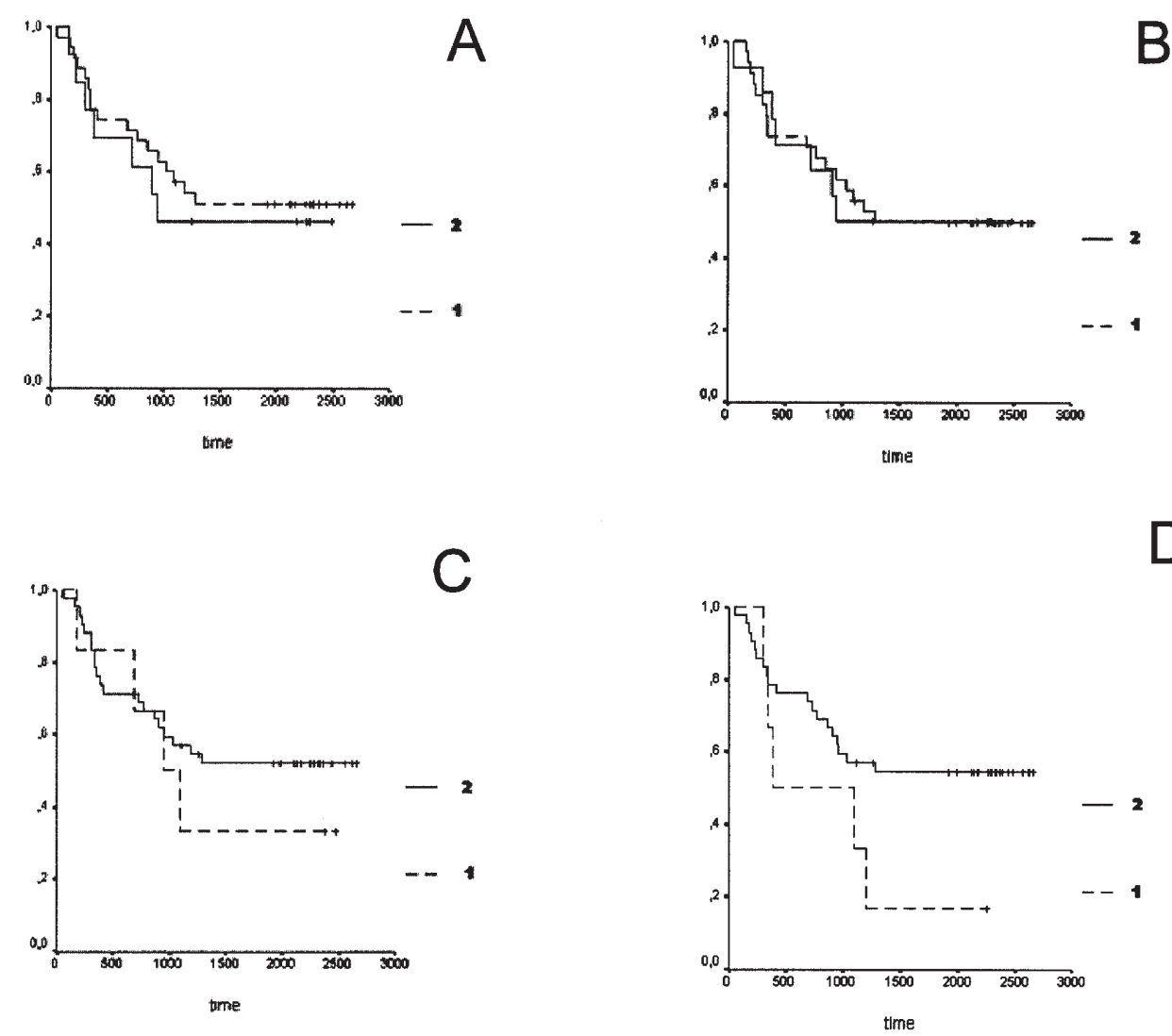

Figure 1. RFS curves for patients expressing CK evaluated by RT-PCR in BM (A), by RT-PCR in PBPC (B), by ICC in BM (C) and by ICC in PBPC (D) respectively. Curve 1: CK positive patients; curve 2: CK negative patients.
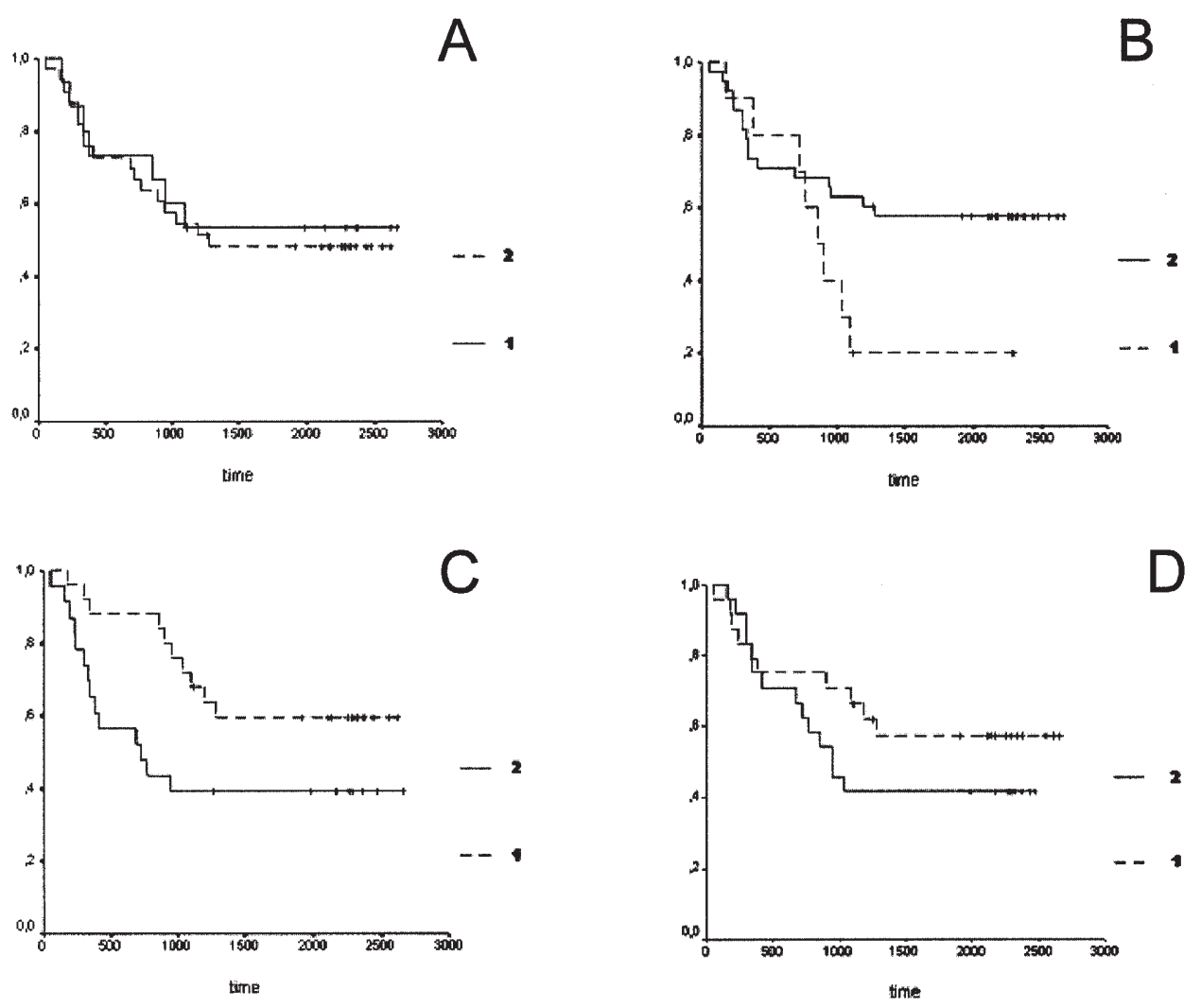

Figure 2. RFS evaluated by RT-PCR in BM of patients MAM+ and MAM- (A, curve 1 and 2); MAS+ and MAS- (C, curve 1 and 2). RFS evaluated by RT-PCR in PBPC of patients MAM+ and MAM- (B, curve 1 and 2); MAS $^{+}$and MAS- (D, curve 1 and 2). 


\section{Discussion}

The TNM classification offers some fairly good chances to evaluate the probability of relapse in patients with large breast tumors and positive nodes. Expression of estrogen/ progesterone receptors, age, and overexpression of oncogenes give other parameters in the decision making after surgery (31).

Unfortunately more than $20 \%$ of low-risk breast cancer patients relapse at 5 years and almost $70 \%$ of high-risk patients develop a metastatic disease within 10 years despite adjuvant chemotherapy. The presence of bone marrow micro-metastatic disease at diagnosis could partially explain this data.

Since 1981, when immunocytochemical techniques for tumoral cell detection were developed, various groups studying different diseases have demonstrated the presence of occult metastasis in the bone marrow and related it with a worse survival (32-37). These metastases were described as tumoral cells expressing epithelial markers and presenting themselves as single or grouped, the latter having the worst prognostic relevance. They can remain long term in a state of dormancy and be difficult to erase by chemotherapy (38). Better results could be reached using monoclonal antibodies, since they continue to express superficial antigens, which could be targeted by immunotherapeutic agents (38). Moreover, BM micrometastatic cells are believed to belong to different clones from those colonizing nodes as they express different superficial molecules as demonstrated by co-immunostaining experiments (39).

Further characterization of these cells will offer a better understanding of their metastatic potential, but it seems possible to explain the failure of the TNM system, at least in part, by the presence at diagnosis of occult tumoral cells in different sites of the body, other than breasts and lymph nodes.

Identification of tumoral cells in the BM is not yet accepted as a standard procedure to be used in order to decide on adjuvant treatments, but for many scientists it represents a valid prognostic tool to be further circumstantiated.

The lack of standardization of the different techniques and the wide numbers of markers that have been used over the years, are two of the major problems explaining the differences in terms of results and reproducibility observed and reported in the past. In particular the use of immunocytochemical techniques leaves a strong subjective bias in identifying different cell sub-populations and scoring the epithelial from the non-epithelial ones. RT-PCR allows a higher percentage of sample positivity, but increases the number of false positives due to pseudo-gene amplification or illegitimate transcription.

Studies on breast cancer have shown that approximately $20-25 \%$ of patients with disseminated disease will have contamination of the PBSC product using routine immunocytochemical analysis (13; Moss TJ, et al, Proc ASCO 16: abs. 90a, 1997). Moss et al performed immunochemistry (ICC) on 133 PBSC apheresis taken from 48 patients to determine the frequency of circulating carcinoma cells: TCC was found in 10\% of cases from approximately 19\% of women (Moss TJ, et al, Proc ASCO 16: abs. 90a, 1997).

The extent of the disease and its sites of metastases seem to correlate with TCC of the hematopoietic support as well as with the number of tumor cells detected (14).
A still unclear number of patients with histologically negative BM have tumor cells detected in the autologous BM or PBSCs used for transplantation $(10,11,16)$.

As reported by the Duke University BM Transplant Program for high-risk primary breast cancer patients, TCC of the BM transplants and even the TC number were associated with a shorter DFS and OS $(17,18)$. In this study, there was no relationship between the TCC of the BM supports and the sites of relapse, suggesting that TCC could be a marker of residual disease prior to HDCT and not directly contributing to relapse.

Moss et al in similar studies on metastatic breast cancer confirmed the data on the poor prognostic value for TCCpositive BM transplants, but failed to obtain significant information about PBSC transplants (Moss TJ, et al, Proc ASCO 16: abs. 90a, 1997).

We introduce a novel approach in evaluating the micrometastatic disease in breast cancer patients, using a contemporary evaluation of a multiple panel of genes, including MAS and MAM and a further step on CK RT-PCR amplification with hybridization of an internal probe to enhance specificity without affecting sensitivity.

Our data confirm that a great proportion of high-risk breast cancer patients present BM micrometastatic disease at diagnosis and many of them, undergoing leukapheresis, present TCC in their autografts. Unfortunately some of such patients have breast cancer cells in their apheresis without histologically evident micrometastases in their bone marrow. We failed to demonstrate any direct relationship between the presence of bone marrow tumoral infiltration and TCC of the apheresis products (data not shown). This could be due to the sensitivity of the methods used, or the limitations of the marrow aspiration procedure, which is limited to a single or double space harvest. Moreover no data are available about the possible mobilization of occult tumoral cells from other sites than BM.

Previously published data showed that tumoral epithelial cells, which are present in the apheresis, are clonogenic and potentially able to cause a relapse (16), but we found no evidence that reinfusing tumoral cells could worsen prognosis. We speculate that a cell, when reinfused in the blood, needs to find an idoneal microenvironment in order to settle and begin to proliferate, and it also needs a permissive immunological status of the recipient.

Unfortunately, we failed to reach statistical significance in the evaluation of prognostic relevance of CK expression as a marker of micrometastatic disease and therefore we could not confirm previously published data. In particular we observed a low level of CK positivity by immunocytochemistry when compared to Braun and colleagues, even though each slide was reviewed by at least two experienced and blinded individuals.

When CK was evaluated by PCR the sensitivity reached very high levels without loosing specificity. In fact by using our hybridization strategy, we were able to eliminate up to $30 \%$ of false positive results depending on erroneing pseudogene amplification or illegitimate transcription. However, $\mathrm{CK}^{+}$vs $\mathrm{CK}^{-}$curves show no difference in RFS advantage probably due to low numbers of events encountered.

MAM seems to be a good marker for specificity, since it is tightly linked to the identification of mammary derived 
cells, but did not for sensitivity, probably because the method used was not able to discriminate between different levels of expression in each sample. Recently, Leone et al showed by real-time PCR that high levels of expression are necessary to obtain an effect on prognosis. In fact, MAMoverexpressing patients showed a statistically significant augmented probability to relapse when compared to low expressing ones (40-41). We suppose to reach that level on leukapheresis products where, after a long follow-up and more events, we demonstrated a direct relationship between MAM expression and relapse.

MAS showed a protective effect on relapse from breast cancer either independently or when plotted against CK. In fact if we consider micrometastatic all those patients who have been found positive for at least one marker in at least one evaluation, those who are positive also for maspin expression have less probability to relapse (data not shown). However the reduced relapse rate appears evident even when MAS is the only positive marker reaching statistical significance $(\mathrm{p}=0.05)$.

Three studies support the hypothesis that MAS works as a protecting factor to epithelial cancer development and metastasis. The first one reports that patients affected by head and neck cancer overexpressing MAS protein experienced a lower incidence of locoregional lymph nodal involvement and had a better prognosis (22). The second shows, in vitro, that recombinant MAS inhibits tumor cell migration and invasion. Additionally, MAS acts as an angiogenesis inhibitor in a rat cornea model and in a xenograft tumor model $(20,21)$. The third study confirms our clinical data on an animal breast cancer model showing that MAS-transfected tumors tended to have tumor encapsulation and less necrosis, which were associated with better prognosis and lower invasiveness (23).

Among metastatic cells, those which express the MAS gene do not acquire neo-angiogenic ability and/or migration capacity. On the other hand MAS down-regulation has been demonstrated to be associated with breast cancer development (42-45). The reverse is also true, since increased levels of MAS expression in vivo protect against tumor progression as demonstrated in two studies using different transgenic mouse models $(46,47)$.

The mechanism of action of MAS seems to be a direct apoptotic effect on breast carcinoma cells since it has been demonstrated that its endogenous expression increases Caspase expression and induces poly-polymerases (PARP) proteolytic inactivation showing an reverse correlation with mutant p53 $(45,48)$.

We could even speculate that MAS protein is in reality produced by other cells than the metastatic ones and patients who have MAS positivity are not metastatic, but protected from the metastatic event.

In conclusion we failed to show a prognostic relevance of all the different markers we used for breast cancer micrometastatic disease evaluation, but the multi-gene panel we created is a possible solution for research into the maximum possible sensitivity and specificity. MAS is supposed to serve as a metastasis-protecting factor with some possible future applications in cancer therapy, since systemic delivery of the MAS gene in a syngeneic tumor model inhibits breast tumor progression (49).

\section{References}

1. Beyer J, Schwella N, Zingsem J, et al: Hematopoietic rescue after high-dose chemotherapy using autologous peripheral-blood progenitor cells or bone marrow: a randomized comparison. J Clin Oncol 13: 1328-1335, 1995.

2. Stadtmauer EA, O'Neill A, Goldstein LJ, et al: Conventionaldose chemotherapy compared with high-dose chemotherapy plus autologous hematopoietic stem-cell transplantation for metastatic breast cancer. N Engl J Med 342: 1069-1076, 2000.

3. Rodenhuis S, Richel DJ, Van der Wall E, et al: Randomised trial of high-dose chemotherapy and haemopoietic progenitorcell support in operable breast cancer with extensive axillary lymph-node involvement. Lancet 15: 515-521, 1998.

4. Mansi JL, Berger U, McDonnel T, et al: The fate of bone marrow micrometastases in patients with primary breast cancer. J Clin Oncol 7: 445-449, 1989.

5. Cote RJ, Rosen PP, Lesser ML, Old LJ and Osborne MP: Prediction of early relapse in patients with operable breast cancer by detection of occult bone marrow micrometastases. J Clin Oncol 9: 1749-1756, 1991.

6. Diel IJ, Kaufmann M, Costa SD, et al: Micrometastatic breast cancer cells in bone marrow at primary surgery: prognostic value in comparison with nodal status. J Natl Cancer Inst 88: 1652-1658, 1996.

7. Braun S, Pantel K, Muller P, et al: Cytokeratin-positive cells in the bone marrow and survival of patients with stage I, II, or III breast cancer. New Engl J Med 342: 525-533, 2000.

8. Fields KK, Elfenbein GJ, Trudeau WL, et al: Clinical significance of bone marrow metastases as detected using the polymerase chain reaction in patients with breast cancer undergoing highdose chemotherapy and autologous bone marrow transplantation. J Clin Oncol 14: 1868-1876, 1996.

9. Pantel K, Schlimok G, Braun S, et al: Differential expression of proliferation-associated molecules in individual micrometastatic carcinoma cells. J Natl Cancer Inst 17: 1419-1424, 1993.

10. Brenner MK, Rill DR, Moen RC, et al: Gene marking and autologous bone marrow transplantation. Ann NY Acad Sci 716: 204-214, 1994.

11. Brugger W, Bross KJ, Glatt M, et al: Mobilization of tumor cells and hematopoietic progenitor cells into peripheral blood of patients with solid tumors. Blood 83: 636-640, 1994.

12. Vredenburgh JJ, Peters WP, Rosner G, et al: Detection of tumor cells in the bone marrow of stage IV breast cancer patients receiving high-dose chemotherapy: the role of induction chemotherapy. Bone Marrow Transplant 16: 815-821, 1995.

13. Bertolini F, Lanza A, Peccatori F, et al: Hematopoietic progenitor cell collection and neoplastic cell contamination in breast cancer patients receiving chemotherapy plus granulocyte-colony stimulating factor (G-CSF) or G-CSF alone for mobilization. Ann Oncol 9: 913-916, 1998.

14. Sharp JC, Kessinger A, Vaughan WP, et al: Detection and clinical significance of minimal TCC of peripheral blood stem cells harvests. Int J Cell Cloning 10 (suppl 1): 92, 1992.

15. Chabannon C, Olivero S, Viret F, et al: Detection of epithelial cells in hematopoietic organs of patients with breast cancer: physiopathological significance and clinical consequences. Acta Haematol 105: 166-171, 2001.

16. Ross A, Cooper BW, Lazarus HM, et al: Detection and viability of tumor cells in peripheral blood stem cell collections from breast cancer patients using immunocytochemical and clonogenic assay techniques. Blood 82: 2605-2610, 1993.

17. Peters WP, Ross M, Vredenburgh JJ, et al: High dose chemotherapy and autologous bone marrow support as consolidation after standard-dose adjuvant therapy for high-risk primary breast cancer. J Clin Oncol 11: 1132-1143, 1993.

18. Vredenburgh JJ, Silva O, Broadwater G, et al: The significance of tumor contamination in the bone marrow from high-risk primary breast cancer patients treated with high-dose chemotherapy with hematopoietic support. Biol Blood Marrow Transpl 3: 91-97, 1997.

19. Funke I and Schraut W: Meta-analyses of studies on bone marrow micrometastases: an independent prognostic impact remains to be substantiated. J Clin Oncol 16: 557-566, 1998.

20. Zhang M, Volpert O, Shi YH and Bouck N: Maspin is an angiogenesis inhibitor. Nat Med 6: 196-199, 2000.

21. Zhu Z, Anisowicz A, Hendrix MJC, et al: Maspin, a serpin with tumor suppressing activity in human mammary epithelial cells. Science 263: 526-529, 1994. 
22. Xia W, Lau YK, Hu MC et al: High tumoral maspin expression is associated with improved survival of patients with oral squamous cell carcinoma. Oncogene 19: 2398-2403, 2000.

23. Shi HY, Zhang V, Liang R, et al: Blocking tumor growth, invasion, and metastasis by maspin in a syngeneic breast cancer model. Cancer Res 61: 6945-6951, 2001.

24. Watson MA and Fleming TP: Mammaglobin, a mammaryspecific member of the uteroglobin gene family, is overexpressed in human breast cancer. Cancer Res 56: 860-865, 1996.

25. Watson MA, Dintzis S, Darrow CM, et al: Mammaglobin expression in primary, metastatic and occult breast cancer. Cancer Res 59: 3028-3031, 1996.

26. Moschinski LC, Trudeau VL, Fields KK and Elfenbein GJ: High sensitivity detection of minimal residual breast carcinoma using the polymerase chain reaction and primers for cytokeratin 19. Diagn Mol Pathol 5: 173-180, 1996.

27. Zach O, Kasparu H, Krieger O, et al: Detection of circulating mammary carcinoma cells in the peripheral blood of breast cancer patients via nested reverse transcriptase polymerase chain reaction assay for mammaglobin mRNA. J Clin Oncol 17: 2015-2019, 1999.

28. Luppi M, Moruzzelli M, Bandieri E, et al: Sensitive detection of circulating breast cancer cells by reverse transcriptase polymerase chain reaction of maspin gene. Ann Oncol 7: 619-624, 1996.

29. Lopez-Guerrero JA, Gilabert PB, Gonzalez EB, et al: Use of reverse transcriptase polymerase chain reaction (RT-PCR) for carcinoma antigen, cytokeratin 19, and maspin in the detection of tumor cells in leukapheresis products from patients with breast cancer: comparison with immunocytochemistry. J Hematol 8: 53-61, 1999 .

30. Franklin WA, Glaspy J, Pflaumer SM, et al: Incidence of tumorcell contamination in leukapheresis products of breast cancer patients mobilized with stem cell factor and granulocyte colonystimulating factor (G-CSF) or with G-CSF alone. Blood 1: 340-347, 1999.

31. Fisher ER: Prognostic and therapeutic significance of pathological features of breast cancer. Natl Cancer Inst Monogr 1: 29-34, 1986.

32. Mansi JI, Berger U, Wilson P, et al: Detection of tumor cells in bone marrow of patients with prostatic carcinoma by immunocytochemical techniques. J Urol 139: 545-546, 1988.

33. Dantas ME, Brown JP, Thomas MR, et al: Detection of melanoma cells in bone marrow using monoclonal antibodies: a comparison of fluorescence activated cell sorting (FACS) and conventional immunofluorescence (IF). Cancer 52: 949-953, 1983.

34. Stahel RA, Mabry M, Skarin AT, et al: Detection of bone marrow metastases in small-cell lung cancer by monoclonal antibody. J Clin Oncol 3: 455-461, 1985.

35. Lindemann F, Schlimok G, Dirschedl P, et al: Prognostic significance of micrometastases tumor cells in bone marrow of colorectal cancer patients. Lancet 340: 685-689, 1992.
36. Dearnaley DP, Sloane JP, Omerod MG, et al: Increased detection of mammary carcinoma cells in marrow smears using antisera to epithelial membrane antigen. Br J Cancer 44: 85-90, 1981

37. Solakoglu O, Maierhofer C, Lahr G, et al: Heterogeneous proliferative potential of occult metastatic cells in bone marrow of patients with solid epithelial tumors. Proc Natl Acad Sci USA 99: 2246-2251, 2002.

38. Kasimir-Bauer S, Mayer S, Bojko P, et al: Survival of tumor cells in stem cell preparations and bone marrow of patients with high-risk or metastatic breast cancer after receiving doseintensive or high-dose chemotherapy. Clin Cancer Res 7: $1582-1589,2001$.

39. Pantel K and Otte M: Occult micrometastasis: enrichment, identification and characterization of single disseminated tumour cells. Semin Cancer Biol 11: 327-337, 2001.

40. Leone F, Perissinotto E, Viale A, et al: Detection of breast cancer cell contamination in leukapheresis product by real-time quantitative polymerase chain reaction. Bone Marrow Transplant 27: 517-523, 2001.

41. Ferrucci PF, Rabascio C, Mazzetta C, et al: Mammaglobin expression in leukapheresis products is a predictive marker of poor prognosis in women with high-risk breast cancer. Clin Cancer Res 10: 6039-6046, 2004.

42. Seftor RE, Seftor EA, Sheng S, et al: Maspin suppresses the invasive phenotype of human breast carcinoma. Cancer Res 58: 5681-5685, 1998.

43. Domann FE, Rice JC, Hendrix MJ, et al: Epigenetic silencing of maspin gene expression in human breast cancers. Int $\mathrm{J}$ Cancer, 85: 805-810, 2000.

44. Zhang $\mathrm{W}$ and Zhang M: Tissue microarray analysis of maspin expression and its reverse correlation with mutant $\mathrm{p} 53$ in various tumors. Int J Oncol 20: 1145-1150, 2002.

45. Maass N, Teffner M, Rosel F, et al: Decline in the expression of the serine proteinase inhibitor maspin is associated with tumor progression in ductal carcinomas of the breast. J Pathol 195: 321-326, 2001.

46. Zhang M, Shi Y, Magit D, et al: Reduced mammary tumor progression in WAP-TAg/WAP-maspin bitransgenic mice. Oncogene 19: 6053-6058, 2000.

47. Reddy KB, McGowen R, Schuger L, et al: Maspin expression inversely correlates with breast tumor progression in MMTV/ TGF-alpha transgenic mouse model. Oncogene 20: 6538-6543, 2001.

48. Jiang N, Meng Y, Zhang S, et al: Maspin sensitizes breast carcinoma cells to induced apoptosis. Oncogene 21:4089-4098, 2002.

49. Shi HY, Liang R, Templeton NS, et al: Inhibition of breast tumor progression by systemic delivery of the maspin gene in a syngeneic tumor model. Mol Ther 5: 755-761, 2002. 